\title{
Numerical Simulations of Compression Properties of SiC/Al Co-continuous Composites
}

\author{
Mingjuan Zhao, Na Li, Longzhi Zhao, and Xiaolan Zhang \\ School of Mechanical and Electrical Engineering, \\ East China Jiao tong University, NanChang 330013, China \\ zhaomingjuan110@163.com, lina19841007@163.com, \\ zhaolongzhi@163.com, Zhangxiaolan85022@163.com
}

\begin{abstract}
The numerical simulations of property of SiC/Al co-continuous composites under compression with ANSYS software were carried out. Based on the simulation results, deformational behavior and distribution of stress and strain fields were analyzed. The effects of compression speed for material property was studied. The results showed that $\mathrm{Al}$ matrix and $\mathrm{SiC}$ reinforcement exhibit different mechanical behavior, large deformation appears inside $\mathrm{Al} \mathrm{ma-}$ trix; the configuration of $\mathrm{SiC}$ has relatively great influence on intensity and distribution of stress within composites, its ultimate stress occurs near the interface of composites; when an external force is exerted on the composites, $\mathrm{Al}$ matrix and $\mathrm{SiC}$ reinforcement restrict each other to prevent from producing the strain; With the increase of loading velocity, the stress of matrix and the strain of reinforcement increase rapidly.
\end{abstract}

Keywords: Composites, Compression properties, ANSYS, Loading velocity.

\section{Introduction}

With the fast development of research and applications technology of metallic matrix composite, the study on its macroscopical performance has to be paid increasing attention. The new composites have metal as a toughening phase and three dimension solid net framework structure as reinforcement, it has become the future trends of metallic matrix composites $[1,2]$. The reinforcement of composites shows three-dimensional network structure, the distribution of holes is usually homogenized, and porosity is controllable [3]. The spatial topological structure has a number of special features, such as, light weight, high specific modulus and specific Strength, abrasion resistance, fatigue resistance, thermal shock resistance, and low coefficient of thermal expansion and so on. It has wide prospect to be applied in various industrial fields, such as aircrafts, automobile, electronics and machinery $[4,5]$. In this article, the compression performance of SiC/Al Co-Continuous Composites was simulated by the finite element analysis software ANSYS.

\section{Creating the Calculation Model of Composite Material}

When the compressive property of composites was simulated, we made some assumptions, an assumed equal division in the holes of $\mathrm{SiC}$ foam ceramics, ribs have 
continuity and size is same; $\mathrm{SiC}$ is absolutely continuous distribution in the composite materials; The physical characteristics of the $\mathrm{SiC}$ and $\mathrm{Al}$ have the properties of isotropy; There is a suitable interfacial bonding between reinforcement and matrix, it belongs to mechanical bond, that is, it depends mainly on mechanical forces of rough surface of reinforcement and contractile force of matrix which wrap up reinforcement and create friction, the forces combine reinforcement and matrix, other impurities is nonexistent at the interface.

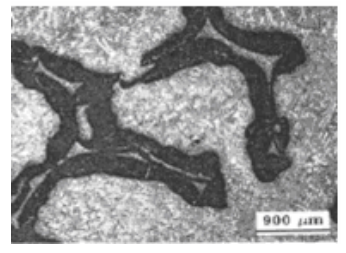

Fig. 1. Macro-appearance of $\mathrm{SiC} / \mathrm{Al}$ Co-Continuous composites

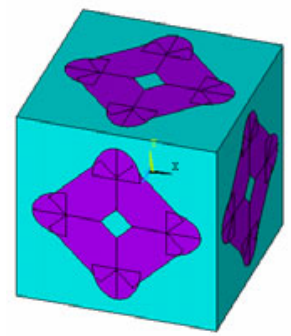

Fig. 2. The simulated model of $\mathrm{SiC} / \mathrm{Al} \mathrm{Co}-\mathrm{Continuous}$ composites

The model of SiC/Al Co-Continuous Composites should be preprocessed before numerical calculation. The primary attribute of $\mathrm{SiC}$ reinforcement is defined, elasticity modulus is $450 \mathrm{Gpa}$, Poisson ratio is 0.17 , density is $2.4 \mathrm{~g} / \mathrm{cm}^{3}$, yield limit is $14 \mathrm{Mpa}$, shear modulus is $192.3 \mathrm{Mpa}$. Unit Type of reinforcement is defined as solid65. The primary attribute of $\mathrm{Al}$ matrix is defined, elasticity modulus is $24 \mathrm{Gpa}$, Poisson ratio is 0.34 , density is $0.6 \mathrm{~g} / \mathrm{cm}^{3}$, yield limit is $7.6 \mathrm{Mpa}$, shear modulus is $80 \mathrm{Mpa}$. Unit Type of matrix is defined as solid45. In order to describe accurately the parameter changes, 16 stacked cells are adopted in numerical simulation. The macro-appearance and simulated model of $\mathrm{SiC} / \mathrm{Al} \mathrm{Co-Continuous} \mathrm{Composites} \mathrm{are} \mathrm{respectively} \mathrm{showed} \mathrm{in}$ Fig.1and Fig.2.

\section{Analysis of Simulation Result}

There is different Mechanical behavior between reinforcement and matrix to metal matrix composite materials. Simulation result of an intercepted cell was analyzed. The isopleths about displacement, stress and strain of the model are showed in Fig 3 8. This paper analyzes entire deformation, difference of elasticity modulus of two constituents cause difference of generated deflection. It can seems from Fig.3, Al matrix in the hole of $\mathrm{SiC}$ foam has taken place large deformation, $\mathrm{SiC}$ plays a supporting role, the stress mainly distributed in $\mathrm{SiC}$ foam. SiC has a clean and distinct outline in Fig.4 and Fig.5. Reinforcement has three-dimensional connected structural characteristic in the composite materials, and reinforcement and matrix have continuity. When an external force is exerted on the composites, $\mathrm{Al}$ matrix and $\mathrm{SiC}$ reinforcement restrict each other to 
prevent from producing the strain; Furthermore, stress distribution has relatively uniform in the center of the interface stress, essentially the stress value is constant; but stress go up tremendously in near the interface boundary, the maximum stress appear in the interfacial boundary or edge.

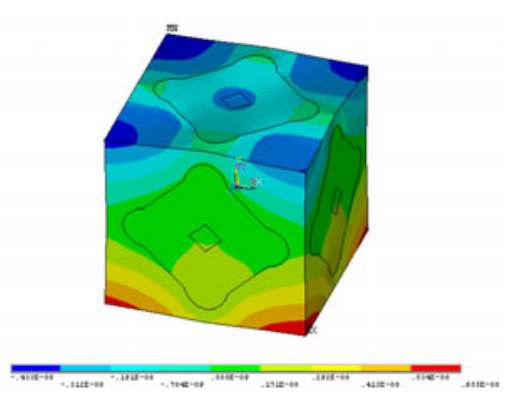

Fig. 3. Isopleths of Y-direction

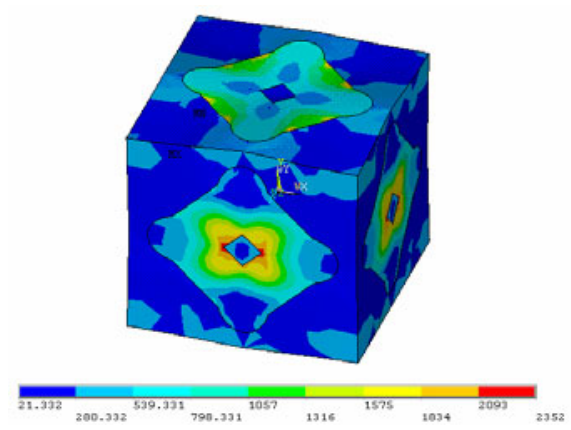

Fig. 4. Isopleths of stress

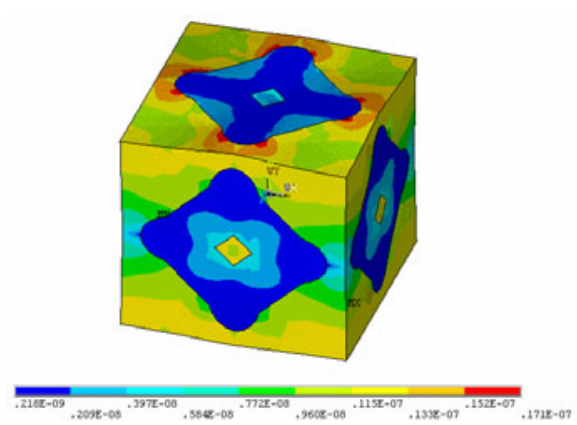

Fig. 5. Isopleths of strain

Additionally, according to the Fig 7 8 which described the isopleths of stress and strain in the different working face, analysis of structure of $\mathrm{SiC}$ foam shows that the structure leads to the distribution of stresses has symmetry, so it has a very significant effect on the distribution and size of stresses. The maximum stress occurred at the interfacial boundary between $\mathrm{SiC}$ reinforcement and $\mathrm{Al}$ matrix. This is also caused by mechanical properties of the reinforcement and matrix, such as, elasticity modulus, yield stress, it is only normal for them to have differences. When an external force is exerted on the composites, the different deformation causes the uneven stress distribution in materials, and furthermore, the deformation behavior of material shows a great difference in near interface of two constituents. This is caused due to the three-dimensional connected structural characteristic, the force on each rib affects other adjacent rib, and stress intensity is related to ribbed-orientation, as well. 


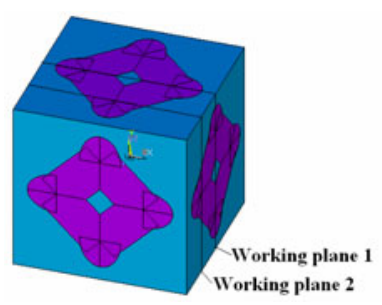

Fig. 6. Diagrammatic sketch of working plane

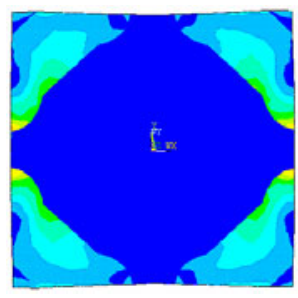

(a)

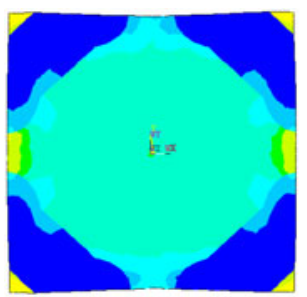

(b)

Fig. 7. Isopleths of stress and strain (Working plane 1)

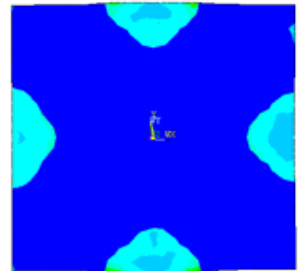

(a)

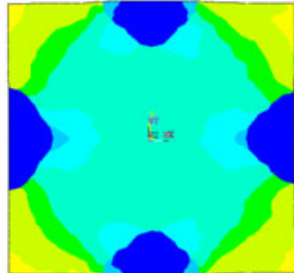

(b)

Fig. 8. Isopleths of stress and strain (Working plane 2)

To sum up, the three-dimensional connected structural characteristic has changed the distribution of stresses of composites, which can restrict the extraneous stretch or compression, and this structural has immensely heightened the tensile strength or compression strength. Moreover, the degree of stress concentration shows that a suitable interfacial bonding can improve the material performance.

\section{Influence of Loading Velocity on Compression Deformation Behavior}

When temperature keep the constant, loading velocity is determined value, the deformation rate-time curve is shown in Fig.9. It can be seen from the graph that as the time is reduced the deformation rate increases, and the trend of deformation reducing slow down, material shows a higher deformability. 


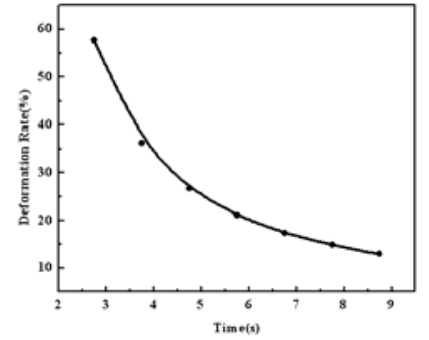

Fig. 9. Deformation rate-Time curve

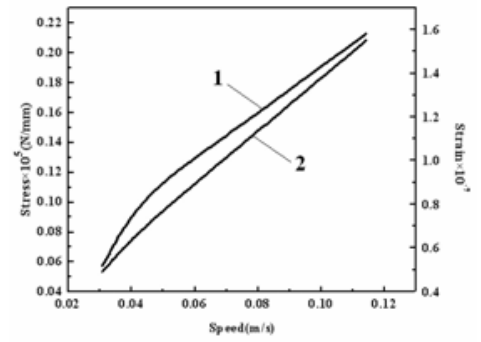

Fig. 10. 1-Stress-Speed curve of reinforcement 2-Strain-Speed curve of matrix

Based on the information provided by the Fig.10, with the fast loading velocity growth, the stress of reinforcement and the strain of matrix are all increase, the strength of composites is also increases. Strength has been mentioned is manifested by material in the static compression. It can be seen in Fig. 10 that value of stress increases slowly with loading velocity raises, microcosmic principle is provided by engineering material can help to explain this, dislocation glide and plastic deformation need time, however, strain rate is too fast and there's not enough time to occur glide, so that materials exhibit rigid. Furthermore, loading velocity is too high, stress suddenly becomes more volatile causes the lower fracture toughness. In the paper perfect state is simulated, assumption has been made in previous paper, but it is difficult to obtain in the material fabrication processes, once the materials have microscopic imperfection, the fast pressure will exacerbate the breakdown speed of composites.

\section{Conclusion}

1) There is different Mechanical behavior between $\mathrm{SiC}$ and $\mathrm{Al}$, difference of elasticity modulus of two constituents occasion difference of generated deflection, Al has taken place large deformation;

2) Due to structural characteristic of $\mathrm{SiC}$, the force on each rib affects other adjacent rib;

3) When an external force is exerted on the composites, $\mathrm{Al}$ and $\mathrm{SiC}$ restrict each other to prevent from producing the strain;

4) With the fast loading velocity growth, the stress of reinforcement and the strain of matrix are all increase; the strength of composites is also increases.

\section{References}

1. Wang, S.H., Geng, H.R., Wang, Y.Z., Sun, B.: The Fabrication Method and Research Progress of the Reticulated Ceramic Reinforcement in Metal Matrix Composites. Materials of Mechanical Engineering 29(12), 1-3 (2005)

2. Zhang, Z.J., Wang, F.C., Yu, X.D., et al.: Research Status of Reticulated Ceramic/Metal Composite. Material Journal 23(1), 20-23 (2009) 
3. Wang, S.R., Geng, H.R., Wang, Y.W., Hui, H.: Model of Compressive Strength of 3DNSRMMCS. Acta Material Composite (23), 7-11 (2006)

4. Qin, S.S.: Metal-matrix Composites for Marine Applications: Development Status and Our Countermeasures. Material Journal 7(10), 68-70 (2003)

5. Yao, J.P., Wang, W.W., Ma, X.S., Yang, M.S.: Wear Behavior of Ceramics Network Composites Fabricated by Die Casting Method. Materials of Mechanical Engineering 39(1), 93-96 (2003) 